\title{
Disease-Related Malnutrition and Sarcopenia as Determinants of Clinical Outcome
}

\author{
Fatuma Meyer Luzia Valentini \\ Neubrandenburg Institute of Evidence-Based Nutrition (NIED), Department of Agriculture and Food Sciences, \\ University of Applied Sciences Neubrandenburg, Neubrandenburg (Mecklenburg-Vorpommern), Germany
}

\section{Keywords}

Undernutrition $\cdot$ Mortality $\cdot$ Morbidity $\cdot$ Prevalence $\cdot$ Costs

\section{Abstract}

Background: Disease-related malnutrition (DRM) and sarcopenia are common complications in chronic or severe disease, with a prevalence in general patient population of 20 $50 \%$ and $0.1-85.4 \%$, respectively. In many patient populations, malnutrition and sarcopenia are present in parallel and often manifest clinically through a combination of decreased nutrient intake, inflammation, and decreased body weight, along with a decrease in muscle mass, strength, and/or physical function, resulting in a clinical condition termed malnutrition-sarcopenia syndrome. Summary: DRM and sarcopenia are associated with increases in all-cause mortality, morbidity, length of hospital stay, and functional impairments (including disabilities and fractures) that lead to a loss of independence and higher costs. Different mortality rates are reported in malnourished patients and well-nourished patients after hospitalization, and higher mortality is the most common complication in patients with sarcopenia. Sarcopenia is a predictor of cancer survival in patients with gastrointestinal, respiratory, and urothelial cancer, and is also related to worse outcomes in patients with liver failure, intestinal insufficiency, and intestinal failure. Length of hospital stay has been found to be longer in DRM and sarcopenic patients in several studies. Prolonged hospitalization due to higher complication rates is often accompanied by demographic changes, resulting in higher hospital and health insurance costs. There are more frequent readmissions by patients with sarcopenia than nonsarcopenic patients. In addition, postoperative complications, duration of hospital stay, and costs increase with advancing sarcopenia stage. A significantly higher complication rate is also reported for DRM, leading to delayed mobilization, lower values in health-related quality of life and more adverse events. DRM is independently associated with poorer clinical outcomes in intensive care unit patients. Muscle dysfunction, as reflected by a decreased handgrip strength, is a well-known consequence of DRM and a good marker of immediate postoperative complications. Most of these outcomes have potential direct or indirect effects on hospital and health care costs, both for the patient and the society at large. Key Messages: Consistent and robust data show DRM and sarcopenia are clinically relevant. They are an increasing problem with relevant medical consequences as well as socioeconomic implications.

(c) 2019 S. Karger AG, Basel

\section{Introduction}

Disease-related malnutrition (DRM) and sarcopenia are common complications in chronic or severe disease. The prevalence of DRM ranges between 20 and 50\% depending on the patient population and the criteria used to determine malnutrition [1]. Several definitions of malnutrition and methods of assessment have been proposed during the last 5 decades; this has produced variable results and hampered comparisons between studies. The most recent descriptive definition explains DRM as a "a state resulting from lack of intake or uptake of nutrition

\section{KARGER}

(C) 2019 S. Karger AG, Basel
Prof. Dr. Luzia Valentini 
that leads to altered body composition (decreased fat-free mass) and body cell mass leading to diminished physical and mental function and impaired clinical outcome from disease" [2].

Operative diagnostic definitions for DRM were proposed by the American Society of Parenteral and Enteral Nutrition (ASPEN) in 2012 [3], the German Society of Nutritional Medicine (DGEM) in 2013/14 [4, 5], and the European Society of Clinical Nutrition and Metabolism in 2015 [6] and 2017 [7]. Despite these definitions, diagnostic steps still varied and disappointment over the failing comparability of results continued. The call for a world-wide unified operative definition increased. So eventually, a global leadership committee with experts from the Essential Fatty Acid Deficiency (EFAD) Conference, ASPEN, the Latin American Society of Parenteral and Enteral Nutrition (Felanpe), and the Parenteral and Enteral Society of Asia (PENSA) was constituted to formulate the global diagnostic criteria for DRM, which were published as the GLIM (Global Leadership Initiative on Malnutrition) criteria in 2018 [8]. GLIM encompasses phenotypic criteria, such as nonvolitional weight loss, low body mass index (BMI), or reduced muscle mass, together with etiologic criteria such as reduced food intake, disease burden, or inflammatory condition.

The term "sarcopenia" was first devised by Irwin Rosenberg in 1989 as the progressive loss of skeletal muscle mass associated with advancing age [9]. In 1997, Rosenberg [10] further elaborated that the term not only describes fundamental changes in body composition but also encompasses function. A variety of sarcopenia definitions are available, but the most generally recognized diagnostic criterion is from the European Working Group on Sarcopenia in Older People (EWGSOP), first published in 2010 [11] and updated as the global definition EWGSOP2 in 2019 (EWGSOP 2) [12]. From these guidelines, conclusions were drawn; the diagnosis of sarcopenia requires a step-wise procedure with subsequent diagnostic testing of low muscle strength, muscle mass, and poor physical performance [12]. Until recently, sarcopenia was considered a geriatric syndrome, but this definition has been expanded to include its development in younger patients due to malnutrition and also the existence of chronic disease and inflammation. In 2016, sarcopenia was identified as an independent condition and added to the International Classification of Disease, 10th revision, Clinical Modification (ICD-10-CM) [13]. A systematic review by Cruz-Jentoft et al. [14] showed the prevalence of sarcopenia using a definition consistent with EWGSOP to be between 1-33\% across different populations (male and female data combined), with a higher prevalence (as to be expected) in settings involving older or acutely ill individuals with an accelerated decline from the 6th decade onwards [15]. Another study as- sessed the prevalence of sarcopenia in a cohort of adult subjects and estimated that the prevalence could range from 0.1 to $85.4 \%$ according to patients' characteristics [16].

In many populations of patients, malnutrition and sarcopenia are present in parallel and often manifest clinically via a combination of disease burden, inflammation, decreased nutrient intake, decreased body weight, altered immune and endocrine functions, and a reduced response to oxidative stress, along with a decrease in muscle mass, strength, and/or physical function [17-19]. Unintentional weight loss is a major sign of malnutrition, which includes a loss of skeletal muscle mass that leads to gradual, progressive muscle wasting. Acute or chronic illness, physical inactivity alone or in concert with an inadequate intake of energy and protein, and inflammation can all hasten the loss of lean body mass and increase the risk of functional impairment [20].

The aim of this report was to evaluate the clinical relevance of DRM and sarcopenia in general, and specifically in patients with intestinal failure (IF).

\section{Results}

\section{Clinical Outcomes of DRM and Sarcopenia}

In general, DRM and sarcopenia are associated with multiple adverse outcomes such as comorbidity, poor physical performance, physical disability, depression, hospitalization, functional decline, falls, an independent increase in all-cause mortality, and a loss of independence when it comes to daily life activities $[16,21-23]$. DRM is currently a major challenge in hospitals, both because of its high prevalence and due to its clinical and economic impact. DRM leads to changes in body composition, with reduced fat and fat-free body mass that often lead to diminished physical and mental function [24]. Impaired immune function, delayed wound-healing, convalescence, and decreased functional status are the main contributors for the enhanced morbidity in DRM [1]. Sarcopenia has been identified as a predictor of poor postoperative clinical outcomes, represents a significant change in health status, and is associated with adverse outcomes such as falls, fractures, functional decline, and increased mortality $[25,26]$. All of these, in turn, negatively affect quality of life and represent a risk factor for other pathologies (as summarized in Fig. 1 by Sanz-Paris et al. $[27,28])$.

Consistent and robust data show that the loss of muscle mass is clinically relevant; although originally described in older people, the emerging evidence suggests that it is of great concern also in the chronically ill nonelderly $[29,30]$. Sarcopenic patients are likely to have worse clinical outcomes than healthy individuals [31]. 


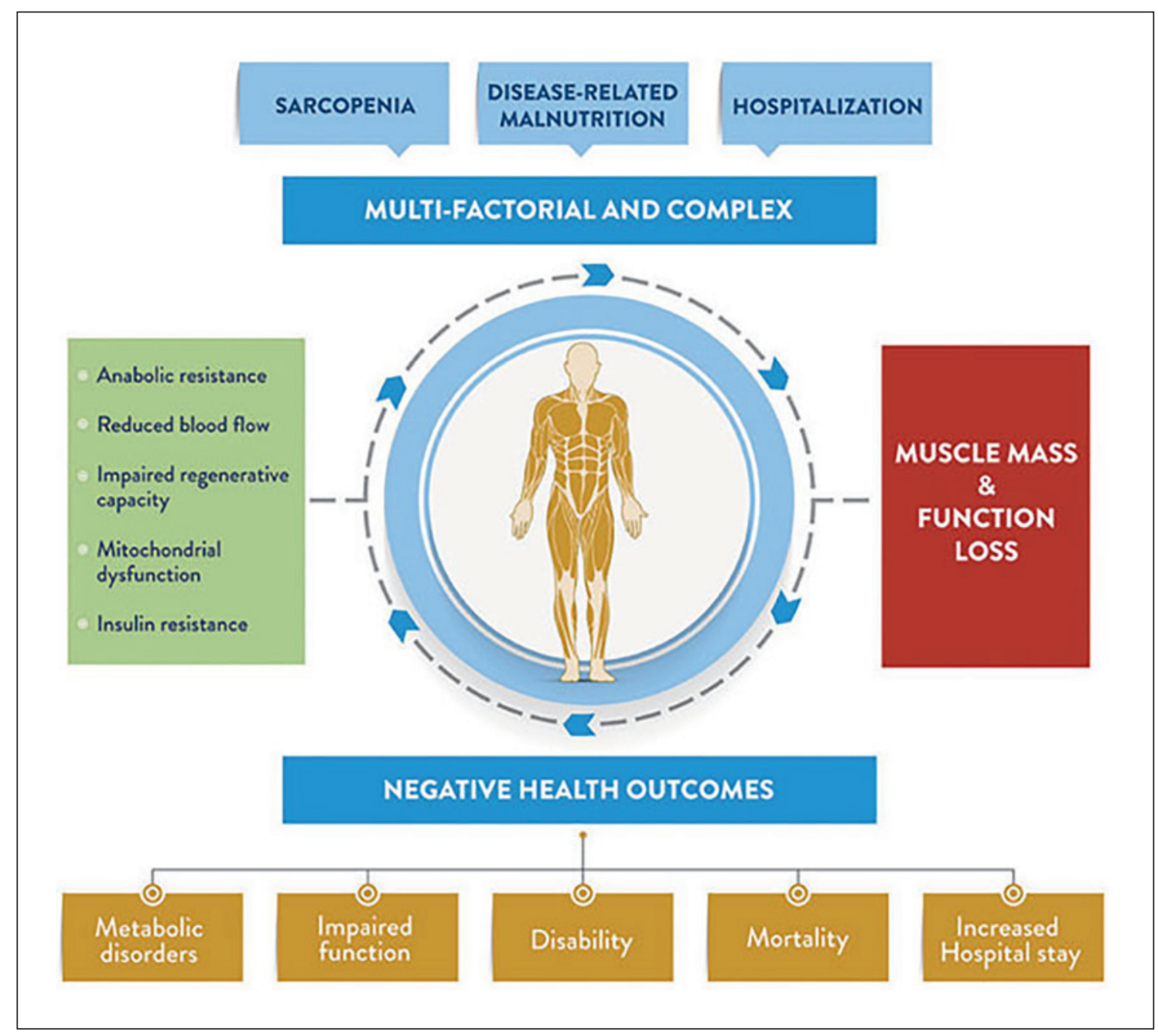

Fig. 1. Common causes and consequences of muscle mass and function loss. Unchanged from [28] (http://creativecommons.org/licenses/by/4.0/).

Regardless of etiology, primary and secondary sarcopenia both increase the likelihood of poor outcomes [30]. In many patient populations, DRM and sarcopenia are present in parallel, and often manifest clinically via a combination of decreased nutrient intake, body weight, muscle mass, strength, and/or even physical function. A clinical syndrome, malnutrition-sarcopenia syndrome (MSS), has been proposed [17].

\section{Mortality}

There is a close relationship between DRM and increased mortality, demonstrated in chronic disease such as liver disease, cancer, terminal renal insufficiency, and chronic obstructive pulmonary disease (COPD), also evident in acute settings [1]. Chronic and acute diseases also have pronounced effects on food intake and metabolism, with increased catabolism which leads to nutrition-related conditions associated with increased morbidity and eventually death [6]. Cederholm et al. [8] reported sig- nificant differences in mortality rates in malnourished (44\%) and well-nourished (18\%) patients after hospitalization. Increased mortality is the most common complication in patients with sarcopenia [30]. While determining the mortality of sarcopenic and nonsarcopenic patients at 6 months, Gariballa and Alessa [32] utilized survival analysis to adjust for known confounders of sarcopenia. They found that nonsarcopenic patients had a significantly lower hazard of death at 6 months than sarcopenic patients (hazard ratio [HR] $0.45 ; 95 \%$ confidence interval [CI] 0.21-0.97]; $p<0.05$ ).

Research indicates that sarcopenia is a predictor of survival in certain diseases. For example, Prado et al. [33] and Fukushima et al. [34] reported in their studies that sarcopenia is a predictor of cancer survival. The presence of sarcopenia was an independent predictor of survival among patients with gastrointestinal and respiratory cancer (HR 4.2; 95\% CI 2.4-7.2; $p<0.0001$ ) and urothelial cancer (HR 3.36; 95\% CI 1.90-6.08; $p<0.001$ ). 
Sarcopenia is also related to worse outcomes among patients with liver failure. Montano-Loza [35] reported a lower median survival ( $34 \pm 11$ vs. $19 \pm 6$ months) and a higher mortality (55 vs. $45 \%$; $p<0.05$ ) of sarcopenic patients with liver failure versus patients who had a normal muscle mass. These results were mirrored by Tandon et al. [36] who observed shorter 1-, 2-, and 3-year survival in sarcopenic versus nonsarcopenic patients with liver failure (sarcopenic: 63,51 , and $51 \%$ vs. nonsarcopenic: 79,74 , and $70 \%$ ). Similarly, 6-month and 1-year survival rates were lower in sarcopenic versus nonsarcopenic patients with cirrhosis (overall: $16 \pm 6$ vs. $28 \pm 3$ months, $p=0.003$; at 6 months: 71 vs. $90 \%, p=0.005$; at 1 year: 53 vs. $83 \%, p=0.005)$ [37].

In a prospective study in China, $\mathrm{Hu}$ et al. [38] investigated the association of MSS with long-term mortality. Of the 453 participants, 14 (3.1\%) had sarcopenia with normal nutrition, 139 (30.7\%) had a risk of malnutrition without sarcopenia, $48(10.6 \%)$ had a risk of malnutrition with sarcopenia, $25(5.5 \%)$ had malnutrition without sarcopenia, and 22 (4.9\%) had MSS at baseline. Compared with nonsarcopenic subjects with normal nutrition, subjects with MSS and subjects with a malnutrition risk and sarcopenia were 4 times more likely to die (HR 4.78 [95\% CI 2.09-10.97] and HR 4.25 [95\% CI 2.22-8.12], respectively), while nonsarcopenic subjects with a malnutrition risk were 2 times more likely to die (HR 2.41 [95\% CI 1.32-4.39]).

Considering sarcopenia as an independent predictor of mortality, Kim et al. [39] showed the risk of death to be 2.99 times and 3.22 times higher in Korean men with sarcopenia identified according to their height- or weightadjusted appendicular skeletal muscle mass (ASM/ $\mathrm{Ht}^{2}$ and ASM/Wt) than nonsarcopenic Korean men, while the HR for death was 5.37 for men with weak leg muscle strength. However, men with a low score for short physical performance battery had a 3.15 times higher risk of death than those with a high score, after adjustment for covariates. The adjusted HR for EWGSOP-defined sarcopenia was 4.00 for ASM/Ht2 and 6.89 for ASM/Wt in men.

\section{Length of Hospital Stay}

DRM and sarcopenia pose an increasing problem, with relevant medical consequences as well as socioeconomic aspects due to the higher rate of complications and the prolonged hospitalization that are often accompanied by demographic changes, and can result in higher hospital and health insurance costs [40]. Given that patients with sarcopenia are at risk for worse hospital-associated outcomes, Gariballa and Alessa [32] in their research observed a longer length of hospital stay of about 13 days for sarcopenic patients versus 9 days for nonsarcopenic patients. There was also frequent readmission of patients with sarcopenia compared with nonsarcopenic patients (55 vs. $32 \% ; p=0.001$ ).

On the other hand, in a sample of patients with colorectal cancer who underwent abdominal computed tomography scan, Lieffers et al. [41] reported a longer hospital stay, a higher frequency of inpatient rehabilitation, and more infectious complications among patients with sarcopenia. Huang et al. [42] investigated the association between sarcopenia and postoperative outcomes in $470 \mathrm{pa}-$ tients, $20.6,10$, and $6.8 \%$ of whom were identified as having presarcopenia, sarcopenia, and severe sarcopenia, respectively. Postoperative complications, duration of hospital stay, and costs increased with advancing sarcopenia stage.

Malnourished surgical patients often have a longer stay in hospital stay, incurring up to $50 \%$ higher costs than those who are not malnourished. Similar findings have also been described in medical patients, particularly older patients [43]. In a study in the UK, compared to patients without sarcopenia, those with sarcopenia had a significantly longer hospital stay (mean $13.4 \pm 8.8$ vs. 9.4 \pm 7 days for nonsarcopenic subjects; $p=0.003$ ) [32]. The association between sarcopenia and hospitalization was also examined by Cawthon et al. [44] and showed a significant association between low muscle density (a risk ratio $[\mathrm{RR}]$ of $1.5 ; 95 \% \mathrm{CI} 1.2-1.7)$ and handgrip strength (RR 1.5; 95\% CI 1.3-1.8) with hospitalization; lean mass, however, was not associated with a risk of hospitalization. In a multicenter observational study on hospitalized patients in Italy, sarcopenia was present in $28 \%$ of the study population; 22 participants died during their hospital stay and 113 died in the year after discharge [45]. Participants with sarcopenia had significantly higher inhospital mortality than those without sarcopenia (6 vs. $2 \% ; p=0.007$ ) and 1-year mortality (26 vs. $14 \%$; $p<$ $0.001)$. After adjusting for potential confounders, sarcopenia was significantly associated with in-hospital mortality (HR 3.45; 95\% CI 1.35-8.86) and 1-year mortality (HR 1.59; 95\% CI 1.10-2.41) [45]. A European-wide clinical study (EuroOOPS) conducted on $>5,000$ patients in 26 hospitals [46], demonstrated higher mortality (12 vs. $1 \%$ ), a significantly longer hospital stay ( 9 vs. 6 days; $p<$ $0.001)$, and a significantly higher rate of complications (odds ratio [OR] 3.47; $p<0.001$ ) for under-/malnourished patients and patients at risk of under-/malnutrition. In a study carried out in Germany [40], the results were similar: patients at risk of malnutrition showed prolonged hospitalization (Nutrition Risk Screening [NRS2002]: 14.5-12.5 days; Mini Nutritional Assessment short-form [MNA-SF]: 13.7-12.4 days; MNA: 13.9-12.3 days; $p<0.05$ ), delayed mobilization (NRS-2002: 2.1-1.7 days; MNA-SF: $1.8-1.7$ days; MNA: 1.9-1.7 days), lower values for health-related quality of life, and more adverse events. 
A systematic review conducted by Lew et al. [22], published in 2017, summarized and explored the association between DRM and poor clinical outcomes among intensive care unit (ICU) patients. They established that DRM diagnosed by nutrition assessment was independently associated with an increased ICU length of hospital stay, ICU readmission, and the risk of hospital mortality. They concluded that DRM is independently associated with poorer clinical outcomes in ICU.

Merli et al. [47] used the Subjective Global Assessment (SGA) in their prospective analysis in 38 consecutive patients undergoing liver transplantation. Their analysis revealed that DRM was identified in 53\% of the cases. Pretransplant nutritional status, hemoglobin concentration, and disease severity were independently associated with the number of episodes of infection during the hospital stay. Malnutrition was the only independent risk factor for the length of stay in the ICU and the total number of days spent in hospital.

\section{Functional Impairment and Morbidity}

Muscle dysfunction is a well-known consequence of DRM; it is reflected by decreased handgrip strength, since this correlates with the loss of total body protein and is known to be a good marker of immediate postoperative complications [1]. In a prospective study in a subacute geriatric care unit [48], almost half of the patients with sarcopenia had a worse prior functional status than those without sarcopenia (Barthel index: 64.2 \pm 22.8 vs. $73.3 \pm 21.8 ; p=0.04$ ) but both groups had a similar functional decline at admission (Barthel index: $24 \pm 15.1$ vs. $28.5 \pm 15.2$; $p=0.1)$ and functional improvement at discharge ( $20.4 \pm 18.3$ vs. $27.4 \pm 21 ; p=$ 0.08). However, Barthel index at discharge remained relatively inferior in sarcopenic patients $(44.2 \pm 26.6 \mathrm{vs}$. $55.9 \pm 26.7 ; p=0.03$ ). After completing a 3-month athome rehabilitation program, no changes in functional capacity were visible among these patients. The Barthel index is an ordinal scale used to measure performance in the activities of daily living. This index is no longer recommended to measure or diagnose sarcopenia in the new EWGSOP 2 criteria [12]. It is interesting to note that, in the following year, according to SánchezRodríguez et al. [49], out of 100 patients, 58 had low muscle mass, which, according to the EWGSOP algorithm, indicates the existence of sarcopenia. No differences were observed in functional capacity between these patients and those with normal muscle mass. When decreased handgrip strength was considered, 47 of these patients met the EWGSOP criteria for severe sarcopenia. In this group, differences in functional capacity were observed at discharge (Barthel index: 45.2 vs. 56.3; $p=0.042$ ) and during a 3 -month follow-up ( 48.3 vs. $59.8 ; p=0.047$ ).

\section{Disability and Fractures}

Sarcopenia is significantly associated with the occurrence of physical disability compared with normal subjects (men and women) [50]. A study in the USA, testing the hypothesis that men and women with low bone mineral density (BMD) and sarcopenia have a higher risk of fracture than those with only one or neither condition, discovered that men with low BMD and sarcopenia had a higher risk of fracture than those with a normal BMD. Their results indicated that sarcopenia alone did not increase fracture risk in either the male cohort with low BMD and sarcopenia nor those with low BMD only. Men with sarcopenia only had a greater risk of fracture than men with a normal BMD and no sarcopenia. On the other hand, the women with sarcopenia only had a greater risk of fracture than those with a normal BMD and no sarcopenia [51].

\section{Economic Implications: Hospital Costs}

Despite the lack of economical assessments, several studies have considered the relationship between sarcopenia and different areas of expenditure such as hospitalization or nursing home admission [18]. Most of these outcomes have potentially direct or indirect effects on hospital and health care costs, both for the patient and the society at large [52]. In 2000, US health care costs directly attributed to sarcopenia were an estimated USD 18.5 billion. This substantial cost is related to the decrease in functional status and also self-autonomy, with a successive rise in falls, disability, and mortality.

Sarcopenia is independently related to hospitalization costs. Direct medical costs attributable to DRM in the USA vary across states, from an annual cost of USD 36-65 per capita [53]. A study at the Johns Hopkins Hospital to assess the relationship between sarcopenia and total hospital costs for patients undergoing operative resection revealed that the presence of sarcopenia was associated with an increase of USD 14,322 in total hospital cost [25]. Patients who presented with sarcopenia demonstrated a higher total hospital cost in the subgroup of patients who developed postoperative complications and in the subgroup that did not develop postoperative complications. Similarly, total hospital cost was higher in patients presenting with sarcopenia regardless of the length of stay for the index admission. According to Sousa et al. [54], sarcopenia increased hospitalization costs by euro 1,240 (95\% CI euro 596-1,887) for patients $<65$ years of age and euro 721 for those $>65$ years. Sarcopenic overweight was related to an increase in hospitalization costs of euro 884 (95\% CI euro 2951,476). In summary, the hospitalization costs are estimated to increase by $58.5 \%$ for patients $<65$ years and $34 \%$ for patients $>65$ years. 
Clinical Outcomes in Intestinal Insufficiency and IF

IF is defined as a state in which the nutritional demands of the body are not met by the gastrointestinal surface [55]. Intestinal insufficiency (II) and IF are characterized by the reduced capacity for processing nutrients and the inability to absorb macro- and micronutrients, and encompass a massive risk of DRM [56]. IF is mainly associated with short bowel syndrome resulting from vascular complications, radiation enteritis effects, Crohn's disease, and intestinal obstruction effects, to mention but a few $[43,55]$. Patients with II or IF are often predisposed to develop secondary sarcopenia [57]. Treatments include long-term home parental nutrition and intestinal transplantation, both of which are costly for the health care system.

In II and IF patients, sarcopenia is common, with recent studies demonstrating a prevalence of $72.7 \%$ in IF patients [15]. A cross-sectional study by Skallerup et al. [56] to investigate the prevalence of sarcopenia and associated risk factors in patients with II and IF showed a high prevalence of sarcopenia (53.1\%; 95\% CI 43.862.2) in the combined sample. However, sarcopenia was more prevalent in patients with IF than in those with II. In a recent study carried out by Oke et al. [58], the majority $(83 \% ; n=56)$ of IF patients had a CT-derived L3 skeletal muscle index (LSMI) below the validated cut-off prior to the start of parental nutrition support, indicating a far greater prevalence of sarcopenia than previously reported.

IF is also associated with a high mortality rate in severely malnourished patients. Clinical outcome was evaluated in a recent Danish retrospective cohort study comprising 77 clinically stable IF patients on home parenteral nutrition for at least 12 months [59]. Although the prevalence of DRM or sarcopenia was not directly assessed in this study, 64 and $60 \%$ of patients had a fatfree mass index (FFMI) and handgrip strength below the reference values and mean FFMI was in the sarcopenia range $\left(14.8 \pm 21 \mathrm{~kg} / \mathrm{m}^{2}\right)$ confirming the previously reported high prevalence of DRM and sarcopenia in IF. Both bioelectrical impedance analysis (BIA)-derived phase angle as well as handgrip strength were negatively associated with the number of readmissions to hospital [59]. Phase angle was also negatively associated with hospital length of stay. FFMI below normal and phase angle below normal were identified as independent risk factors for mortality during the median follow-up period of 24 months (HR 3.9 [95\% CI 1.1-4.1], $p=0.04$ and HR 5.3 [95\% CI 1.6-17.5], $p=0.007$, respectively).

Cost effective estimations were carried out by Roskott et al. [60] in a simulated disease course of irreversible IF with home parenteral nutrition versus intestinal transplantation. Intestinal transplantation slightly improved the survival of patients compared with home parenteral nutrition (14.9 vs. 14.6 years) at an additional cost of euro 19,529 per life-year gained.

\section{Practical Implications: Similarities and Differences of DRM and Sarcopenia}

The Results section pointed towards a seemingly close relationship between DRM and sarcopenia, resulting in the newly coined term, MSS. For practical reasons, one might ask if it is necessary to diagnose both conditions, or which of the two is more important and should be screened and diagnosed in daily clinical practice. To answer this question, we indicate in detail the similarities and differences in the diagnosis of malnutrition (GLIM [8]) and sarcopenia (EWGSOP 2 [12]) according to the new worldwide definitions. Both follow a 2-step procedure, with an initial screening of all patients, followed by a more detailed diagnostic procedure in patients with positive screening results.

\section{Screening for DRM and Sarcopenia in All Patients}

Screening of DRM can be done by any validated screening questionnaire, such as malnutrition screening tool (MUST [61]) and MNA-SF [62], among others. In continental Europe, the NRS-2002 is most often used and is well established [63]. Thus, we focused on the NRS-2002 in the following comparison. Sarcopenia screening can be done using only one single screening questionnaire, in this case the SARC-F [64], with the acronym combining the initial letters of the 5 screening questions. Figure 2 compares both screening procedures for GLIM and EWGSOP 2. It is quite evident that the questions of these screening procedures do not overlap. NRS-2002 focuses on nutrition and underlying disease whereas SARC-F concentrates on muscle strength and physical function in a Barthel index-like fashion.

\section{Diagnosis of DRM and Sarcopenia}

Figure 3 compares the algorithm of DRM diagnosis according to the GLIM criteria and sarcopenia diagnosis according to the EWGSOP 2 criteria. Both algorithms start after a positive risk screening and encompass the same steps, i.e., diagnostic assessment, diagnosis, and severity grading. However, the diagnostic criteria are markedly different. Whereas GLIM addresses the whole panel of malnutrition symptoms, such as nonvolitional weight loss, low body mass, reduced food intake, disease burden, inflammation, and reduced muscle mass, sarcopenia focuses solely on muscle strength, muscle mass, and function. 


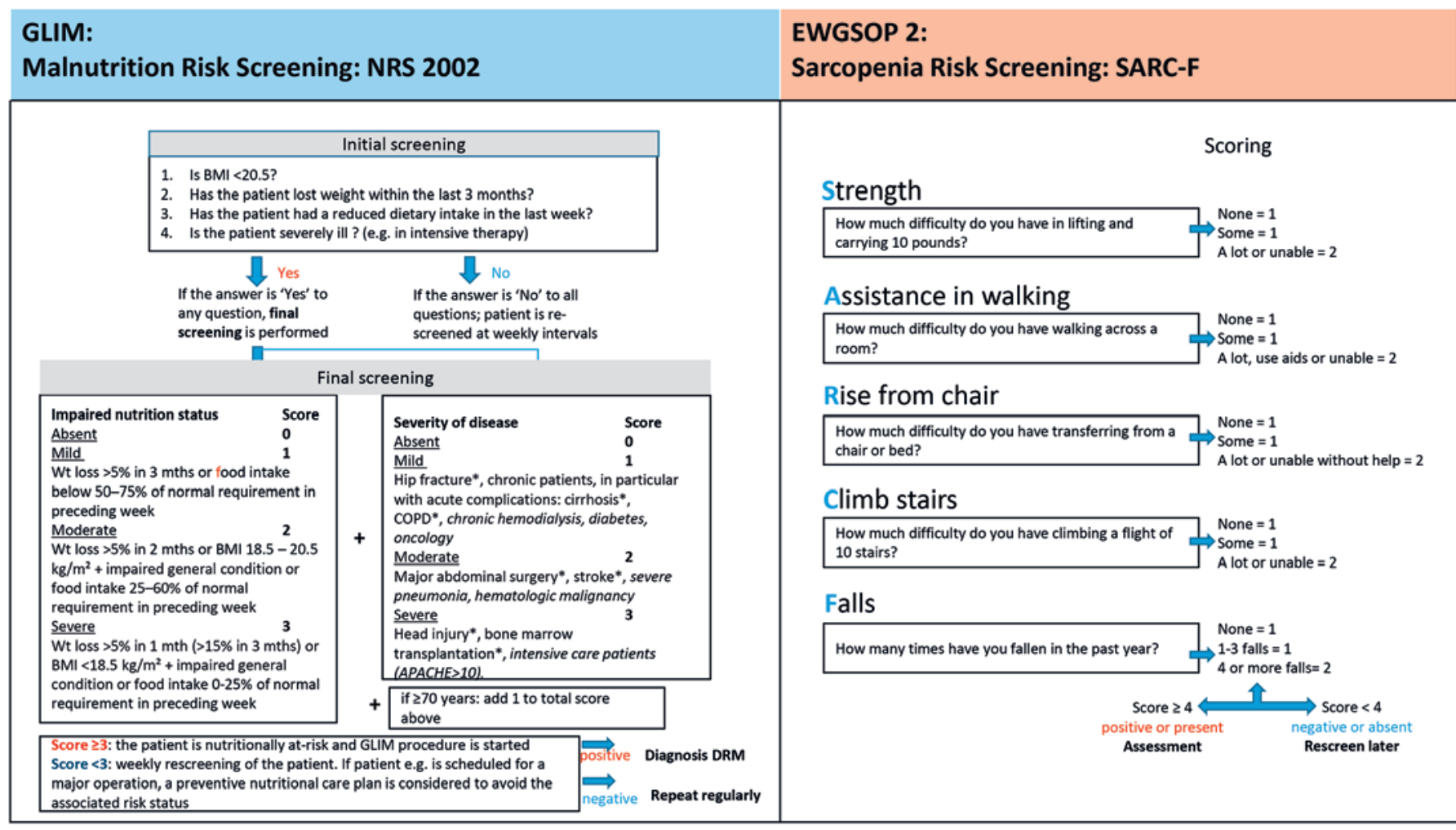

Fig. 2. Comparison of the screening for malnutrition as part of GLIM (NRS-2002 [63]) and sarcopenia as part of EWGSOP 2 (SARC-F [65]). NRS-2002 is based on an interpretation of available randomized clinical trials. The asterisk indicates that a trial directly supports the categorization of patients with that diagnosis.
Diagnoses shown in italics are based on the prototypes given below. Wt, weight; $\mathrm{mth}(\mathrm{s})$, month(s). Positive screening results lead the GLIM algorithm. SARC-F shows 5 questions focusing on the rough estimates of muscle strength and physical performance.

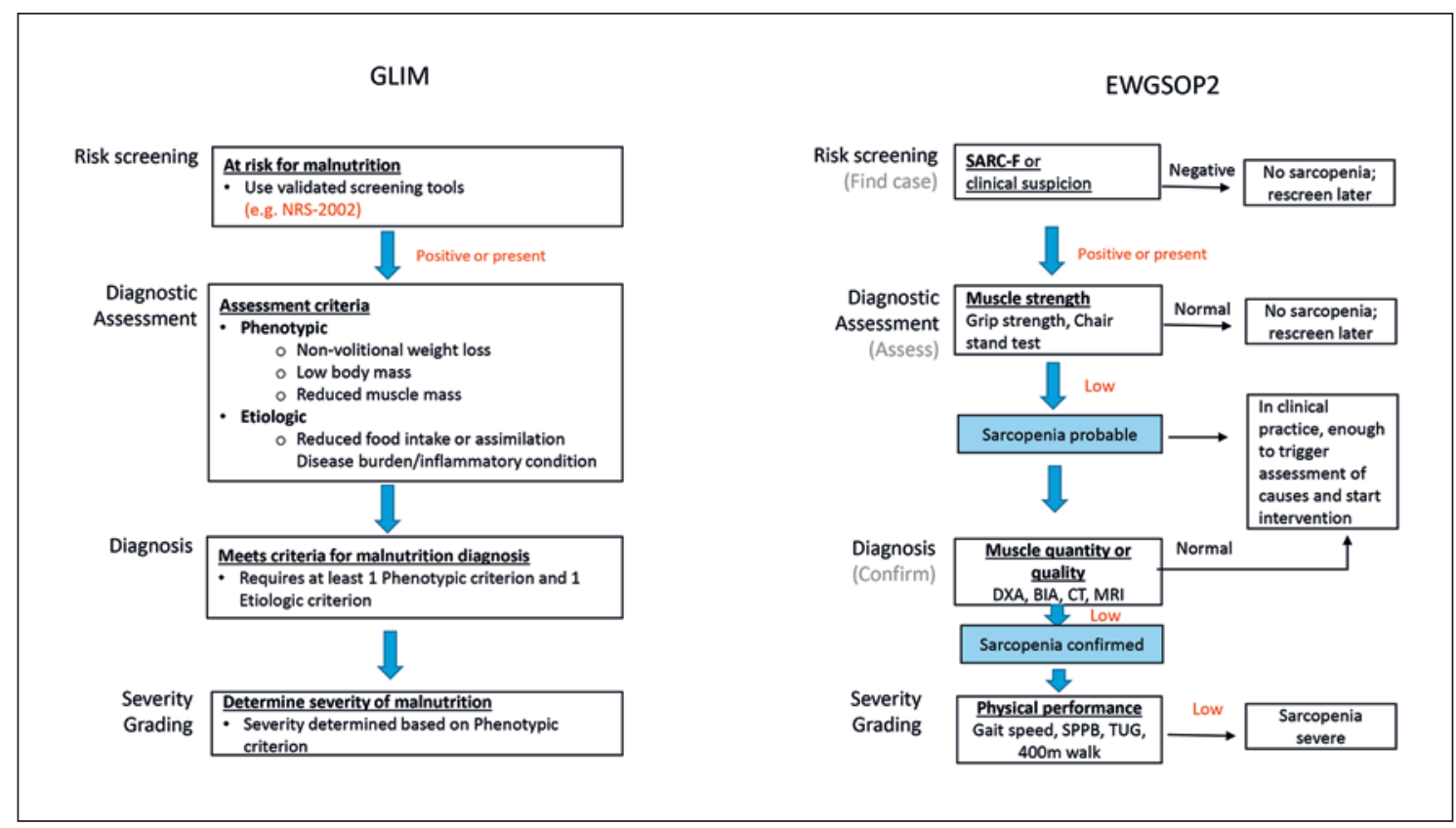

Fig. 3. Comparison of diagnostic criteria and respective algorithms for malnutrition (GLIM) and sarcopenia (EWGSOP 2) according to Cederholm et al. [8] and Cruz-Jentoft et al. [12]. DXA, dual-energy X-ray absorptiometry; BIA, bioelectrical impedance analysis; CT, computed tomography; MRI, magnetic resonance imaging (MRI); SPPB, short physical performance battery; TUG, timed "up and go" test. 


\section{Practical Consequences}

The prospective study of $\mathrm{Hu}$ et al. [38] clearly shows that only $3.1 \%$ of well-nourished patients are sarcopenic, but $>50 \%$ of patients are at a risk of malnutrition or are malnourished and $15 \%$ have a combined risk of malnutrition/sarcopenia. This points toward the greater importance of malnutrition diagnoses versus sarcopenia diagnoses in daily clinical practice.

So far, no official recommendations exist on how to combine DRM and sarcopenia screening and diagnosis for daily practice. However, for general internal or surgical patients, it would seem reasonable to start with malnutrition screening and diagnosis as recommended, with the addition of sarcopenia procedures only when signs of depleted muscle mass or muscle strength are evident during the malnutrition assessment procedure.

\section{Conclusion}

DRM is common and has a wide spectrum of effects, not only with regard to physiological functions; it is also associated with increased rates of mortality and morbid- ity in hospitalized patients. This has a ripple effect on overall health care costs [43]. Depleted muscle mass is associated with infectious complications, a prolonged duration of mechanical ventilation, longer hospitalization, readmission to the hospital, a greater need for rehabilitation care after hospital discharge, and higher mortality among patients identified as sarcopenic [30]. While a large body of work supports the fact that DRM and sarcopenia are associated with poor clinical outcomes, further efforts to standardize the modalities to diagnose DRM and sarcopenia for clinical research and clinical practice may help to strengthen future prospective investigations beneficial for determining effective prevention and treatment strategies to minimize the detrimental effects of DRM and sarcopenia [15].

\section{Disclosure Statement}

The authors declare there are no conflicts of interest.

\section{References}

1 Norman K, Pichard C, Lochs H, Pirlich M. Prognostic impact of disease-related malnutrition. Clin Nutr. 2008;27(1):5-15.

2 Van Bokhorst-de van der Schueren MA, Soeters PB, Reijven PL, Allison SP, Kondrup J. Diagnosis of malnutrition - screening and assessment. In: Sobotka L, editor. Basics in Clinical Nutrition. 4th ed. Prague: Galén; 2011. p. 21-32

3 White JV, Guenter P, Jensen G, Malone A, Schofield M; Academy of Nutrition and Dietetics Malnutrition Work Group; A.S.P.E.N. Malnutrition Task Force; A.S.P.E.N. Board of Directors. Consensus statement of the Academy of Nutrition and Dietetics/American Society for Parenteral and Enteral Nutrition: characteristics recommended for the identification and documentation of adult malnutrition (undernutrition). J Acad Nutr Diet. 2012 May;112(5):730-8

4 Valentini L, Volkert D, Schütz T, Ockenga J, Pirlich M, Druml W, et al. Leitlinie der Deutschen Gesellschaft für Ernährungsmedizin (DGEM). Aktuel Ernahrungsmed. 2013; 38(02):97-111.

5 Valentini L, Volkert D, Schütz T, Ockenga J, Pirlich M, Druml W, et al. Suggestions for terminology in clinical nutrition. e-SPEN J. 2014;9(2):e97-e108.

6 Cederholm T, Bosaeus I, Barazzoni R, Bauer J, Van Gossum A, Klek S, et al. Diagnostic criteria for malnutrition - An ESPEN Consensus Statement. Clin Nutr. 2015;34(3):335-40.

7 Cederholm T, Barazzoni R, Austin P, Ballmer P, Biolo G, Bischoff SC, et al. ESPEN guidelines on definitions and terminology of clinical nutrition. Clin Nutr. 2017;36(1):49-64.
8 Cederholm T, Jensen GL, Correia M, Gonzalez MC, Fukushima R, Higashiguchi T, et al. GLIM criteria for the diagnosis of malnutrition - a consensus report from the global clinical nutrition community. Clin Nutr. 2019;38:1-9.

9 Rosenberg I. Summary comments. Am J Clin Nutr. 1989;50(5):1231-3.

10 Rosenberg IH. Sarcopenia: origins and clinical relevance. J Nutr. 1997 May;127(5 Suppl): 990S-1S.

11 Cruz-Jentoft AJ, Baeyens JP, Bauer JM, Boirie Y, Cederholm T, Landi F, et al. Sarcopenia: European consensus on definition and diagnosis: report of the European Working Group on Sarcopenia in Older People. Age Ageing. 2010;39(4):412-23.

12 Cruz-Jentoft AJ, Bahat G, Bauer J, Boirie Y, Bruyère $\mathrm{O}$, Cederholm $\mathrm{T}$, et al.; Writing Group for the European Working Group on Sarcopenia in Older People 2 (EWGSOP2), and the Extended Group for EWGSOP2. Sarcopenia: revised European consensus on definition and diagnosis. Age Ageing. 2019 Jan; 48(1):16-31.

13 Cao L, Morley JE. Sarcopenia Is Recognized as an Independent Condition by an International Classification of Disease, Tenth Revision, Clinical Modification (ICD-10-CM) Code. J Am Med Dir Assoc. 2016 Aug;17(8): 675-7.

14 Cruz-Jentoft AJ, Landi F, Schneider SM, Zúñiga C, Arai $\mathrm{H}$, Boirie $\mathrm{Y}$, et al. Prevalence of and interventions for sarcopenia in ageing adults: a systematic review. Report of the International Sarcopenia Initiative (EWGSOP and IWGS). Age Ageing. 2014 Nov;43(6): 748-59.
15 Han A, Bokshan S, Marcaccio S, DePasse J, Daniels AJJocm. Diagnostic criteria and clinical outcomes in sarcopenia research: a literature review. J Clin Med. 2018;7(4):70.

16 Beaudart C, Rizzoli R, Bruyère O, Reginster JY, Biver E. Sarcopenia: burden and challenges for public health. Arch Public Health. 2014 Dec;72(1):45

17 Vandewoude MF, Alish CJ, Sauer AC, Hegazi RA. Malnutrition-sarcopenia syndrome: is this the future of nutrition screening and assessment for older adults? J Aging Res. 2012; 2012:651570.

18 Wijarnpreecha K, Panjawatanan P, Thongprayoon $\mathrm{C}$, Jaruvongvanich $\mathrm{V}$, Ungprasert $\mathrm{P}$. Sarcopenia and risk of nonalcoholic fatty liver disease: a meta-analysis. Saudi J Gastroenterol. 2018;24(1):12.

19 Greig C. Nutritional approaches to the management of sarcopenia. J Nutr Bull. 2013; 38(3):344-8.

20 Jacobsen EL, Brovold T, Bergland A, Bye A. Prevalence of factors associated with malnutrition among acute geriatric patients in Norway: a cross-sectional study. BMJ Open. 2016; 6(9):e011512.

21 Tsekoura M, Kastrinis A, Katsoulaki M, Billis E, Gliatis J. Sarcopenia and Its Impact on Quality of Life. GeNeDis. Springer; 2016. p. 213-8.

22 Lew CC, Yandell R, Fraser RJ, Chua AP, Chong MF, Miller M. Association between Malnutrition and Clinical Outcomes in the Intensive Care Unit: A Systematic Review. J Parenter Enteral Nutr. 2017 Jul;41(5):744-58. 
23 De Spiegeleer A, Petrovic M, Boeckxstaens P, Van Den Noortgate N. Treating sarcopenia in clinical practice: where are we now? Acta Clin Belg. 2016 Aug;71(4):197-205.

24 Deutz NE, Ashurst I, Ballesteros MD, Bear DE, Cruz-Jentoft AJ, Genton L, et al. The Underappreciated Role of Low Muscle Mass in the Management of Malnutrition. J Am Med Dir Assoc 2019;20(1):22-7.

25 Gani F, Buettner S, Margonis GA, Sasaki K, Wagner D, Kim Y, et al. Sarcopenia predicts costs among patients undergoing major abdominal operations. Surgery. 2016;160(5) 1162-71.

26 Arango-Lopera VE, Arroyo P, Gutiérrez-Robledo LM, Perez-Zepeda MU, Cesari M. Mortality as an adverse outcome of sarcopenia. J Nutr Health Aging. 2013;17:259-62.

27 Sgrò P, Sansone M, Sansone A, Sabatini S, Borrione P, Romanelli F, et al. Physical exercise, nutrition and hormones: three pillars to fight sarcopenia AU. Aging Male. 2019;22: 75-88.

28 Sanz-Paris A, Camprubi-Robles M, LopezPedrosa JM, Pereira SL, Rueda R, BallesterosPomar MD, et al. Role of Oral Nutritional Supplements Enriched with B-hydroxy-BMethylbutyrate in Maintaining Muscle Function and Improving Clinical Outcomes in Various Clinical Settings. J Nutr Health Aging. 2018;22(6):664-75.

29 Laviano A, Gori C, Rianda S. Sarcopenia and nutrition. Adv Food Nutr Res. 2014;71:10136.

30 Peterson SJ, Braunschweig CA. Prevalence of Sarcopenia and Associated Outcomes in the Clinical Setting. Nutr Clin Pract. 2016;31(1): 40-8.

31 Palus S, Springer JI, Doehner W, von Haehling S, Anker M, Anker SD, et al. Models of sarcopenia: short review. Int J Cardiol. 2017 Jul;238:19-21.

32 Gariballa S, Alessa AJ. Sarcopenia: prevalence and prognostic significance in hospitalized patients. Clin Nutr. 2013;32(5):772-6.

33 Prado CM, Lieffers JR, McCargar LJ, Reiman T, Sawyer MB, Martin L, et al. Prevalence and clinical implications of sarcopenic obesity in patients with solid tumours of the respiratory and gastrointestinal tracts: a populationbased study. Lancet Oncol. 2008 Jul;9(7):629_ 35 .

34 Fukushima H, Yokoyama M, Nakanishi Y, Tobisu K, et al. Sarcopenia as a prognostic biomarker of advanced urothelial carcinoma. PLos One. 2015;10(1):e0115895.

35 Montano-Loza AJ. Clinical relevance of sarcopenia in patients with cirrhosis. World J Gastroenterol. 2014 Jul;20(25):8061-71.

36 Tandon P, Ney M, Irwin I, Ma MM, Gramlich $\mathrm{L}$, Bain VG, et al. Severe muscle depletion in patients on the liver transplant wait list: its prevalence and independent prognostic value. Liver Transplant. 2012;18(10):1209-16.

37 Meza-Junco J, Montano-Loza AJ, Baracos VE, Prado CM, Bain VG, Beaumont C, et al. Sarcopenia as a prognostic index of nutritional status in concurrent cirrhosis and hepatocellular carcinoma. J Clin Gastroenterol. 2013;47(10):861-70.
$38 \mathrm{Hu} \mathrm{X}$, Zhang L, Wang H, Hao Q, Dong B, Yang M. Malnutrition-sarcopenia syndrome predicts mortality in hospitalized older patients. Sci Rep. 2017 Jun;7(1):3171.

39 Kim JH, Lim S, Choi SH, Kim KM, Yoon JW, Kim KW, et al. Sarcopenia: an independent predictor of mortality in community-dwelling older Korean men. J Gerontol A Biol Sci Med Sci. 2014;69(10):1244-52.

40 Ihle C, Weiß C, Blumenstock G, Stöckle U, Ochs BG, Bahrs C, et al. Interview based malnutrition assessment can predict adverse events within 6 months after primary and revision arthroplasty - a prospective observational study of 351 patients. BMC Musculoskelet Dis. 2018;19(1):83.

41 Lieffers J, Bathe O, Fassbender K, Winget M, Baracos VJBjoc. Sarcopenia is associated with postoperative infection and delayed recovery from colorectal cancer resection surgery. Br J Cancer. 2012;107(6):931.

42 Huang D-D, Zhou C-J, Wang S-L, Mao S-T, Zhou X-Y, Lou N, et al. Impact of different sarcopenia stages on the postoperative outcomes after radical gastrectomy for gastric cancer. Surgery. 2017;161(3):680-93.

43 Saunders J, Smith T. Malnutrition: causes and consequences. Clin Med (Lond). 2010 Dec; 10(6):624-7.

44 Cawthon PM, Fox KM, Gandra SR, Delmonico MJ, Chiou CF, Anthony MS, et al. Do muscle mass, muscle density, strength, and physical function similarly influence risk of hospitalization in older adults? J Am Geriatric Soc. 2009;57(8):1411-9.

45 Vetrano DL, Landi F, Volpato S, Corsonello A, Meloni E, Bernabei R, et al. Association of sarcopenia with short-and long-term mortality in older adults admitted to acute care wards: results from the CRIME study. J Gerontol A Biol Sci Med Sci. 2014;69(9): 1154-61.

46 Sorensen J, Kondrup J, Prokopowicz J, Schiesser M, Krähenbühl L, Meier R, et al. EuroOOPS: an international, multicentre study to implement nutritional risk screening and evaluate clinical outcome. Clin Nutr. 2008;27(3):340-9.

47 Merli M, Giusto M, Gentili F, Novelli G, Ferretti G, Riggio O, et al. Nutritional status: its influence on the outcome of patients undergoing liver transplantation. Liver Int. 2010; 30(2):208-14.

48 Sánchez-Rodríguez D, Marco E, Miralles R, Fayos M, Mojal S, Alvarado M, et al. Sarcopenia, physical rehabilitation and functional outcomes of patients in a subacute geriatric care unit. Arch Gerontol Geriatr. 2014 JulAug;59(1):39-43.

49 Sánchez-Rodríguez D, Marco E, Miralles R, Guillén-Solà A, Vázquez-Ibar O, Escalada F, et al. Does gait speed contribute to sarcopenia case-finding in a postacute rehabilitation setting? Arch Gerontol Geriatr. 2015 Sep-Oct; 61(2):176-81

50 Tanimoto Y, Watanabe M, Sun W, Tanimoto K, Shishikura K, Sugiura Y, et al. Association of sarcopenia with functional decline in community-dwelling elderly subjects in Japan. Geriatr Gerontol Int. 2013;13(4):958-63.
51 Chalhoub D, Cawthon PM, Ensrud KE, Stefanick ML, Kado DM, Boudreau R, et al. Risk of nonspine fractures in older adults with sarcopenia, low bone mass, or both. J Am Geriatr Soc. 2015;63(9): 1733-40.

52 Bruyère $\mathrm{O}$, Beaudart $\mathrm{C}$, Ethgen $\mathrm{O}$, Reginster J-Y, Locquet M. The health economics burden of sarcopenia: a systematic review. Maturitas. 2019;119:61-9.

53 Goates S, Du K, Braunschweig CA, Arensberg MBJPo. Economic burden of disease-associated malnutrition at the state level. PLos One. 2016;11(9):e0161833.

54 Sousa A, Guerra R, Fonseca I, Pichel F, Ferreira S, Amaral TJEjocn. Financial impact of sarcopenia on hospitalization costs. Eur J Clin Nutr. 2016;70(9):1046.

55 Kappus M, Diamond S, Hurt RT, Martindale R. Intestinal failure: new definition and clinical implications. J. Curr Gastroenterol Rep. 2016 Sep; 18(9): 48.

56 Skallerup A, Nygaard L, Olesen SS, Kohler M, Vinter-Jensen L, Rasmussen HH. The prevalence of sarcopenia is markedly increased in patients with intestinal failure and associates with several risk factors. Clin Nutr. 2018;37(6 Pt A):2029-35.

57 Thibault R, Pichard C. The evaluation of body composition: a useful tool for clinical practice. Ann Nutr Metab. 2012;60(1):6-16.

58 Oke SM, Rye B, Malietzis G, Baldwin-Cleland R, Bottle A, Gabe SM, et al. Survival and CT defined sarcopenia in patients with intestinal failure on home parenteral support. Clin Nutr. 2019 Mar 19. doi: 10.1016/j.clnu. 2019.03.015. Epub ahead of print

59 Køhler M, Olesen SS, Rasmussen HH. Body composition predicts clinical outcome in patients with intestinal failure on long-term home parenteral nutrition. Clin Nutr ESPEN. 2018;28:193-200.

60 Roskott AM, Groen H, Rings EH, Haveman JW, Ploeg RJ, Serlie MJ, et al. Cost-effectiveness of intestinal transplantation for adult patients with intestinal failure: a simulation study. Am J Clin Nutr. 2015 Jan;101(1):7986.

61 Stratton RJ, Hackston A, Longmore D, Dixon $\mathrm{R}$, Price S, Stroud M, et al. Malnutrition in hospital outpatients and inpatients: prevalence, concurrent validity and ease of use of the 'malnutrition universal screening tool' ('MUST') for adults. Br J Nutr. 2004 Nov; 92(5):799-808.

62 Rubenstein LZ, Harker JO, Salvà A, Guigoz Y, Vellas B. Screening for Undernutrition in $\mathrm{Ge}$ riatric Practice: Developing the Short-Form Mini-Nutritional Assessment (MNA-SF). Gerontol Series A. 2001;56(6):M366-M72.

63 Kondrup J, Rasmussen HH, Hamberg O, Stanga Z. Nutritional risk screening (NRS 2002): a new method based on an analysis of controlled clinical trials. Clin Nutr. 2003; 22(3):321-36.

64 Woo J. Sarcopenia. Clin Geriatr Med. 2017; 33(3):305-14.

65 Malmstrom TK, Morley JE. SARC-F: a simple questionnaire to rapidly diagnose sarcopenia. J Am Med Dir Assoc. 2013 Aug;14(8):531-2. 